\title{
Optimisation Multiobjectif du profil NACA0012 basée sur l'algorithme génétique de Tri Non-dominé
}

\section{Multiobjective Optimization of NACA0012 Airfoil based Non-dominated}

\author{
Sorting Genetic Algorithm
}

\author{
Soufiane Elouardi ${ }^{1}$, Rabii El Maani ${ }^{2}$, Bouchaib Radi ${ }^{3}$ \\ ${ }^{1}$ LIMII, FST Settat, Route de Casablanca, Settat, Maroc, so.elouardi@uhp.ac.ma \\ ${ }^{2}$ LSMI, ENSAM Meknès, Route de Casablanca, Marjane 2, Maroc, elmaani.rabi3@gmail.com \\ ${ }^{3}$ LIMII, FST Settat, Route de Casablanca, Settat, Maroc, bouchaib.radi@yahoo.fr
}

\begin{abstract}
RÉSUMÉ. Les techniques d'optimisation multidisciplinaires sont de plus en plus appliquées dans le domaine de l'aérodynamique en raison du développement rapide des ordinateurs à haute performance, des méthodes numériques et des algorithmes d'optimisation. Ces techniques, associées à la dynamique numérique des fluides (CFD), qui vise à intégrer des relations et des algorithmes mathématiques pour analyser et résoudre les problèmes d'écoulement des fluides, impliquent l'utilisation de ces méthodes et algorithmes numériques pour améliorer les solutions d'écoulement des fluides. L'analyse CFD d'un profil aérodynamique détermine sa capacité en produisant des résultats tels que les forces de portance et de traînée, et l'application d'un algorithme d'optimisation implique la manipulation de ces performances aérodynamiques en fonction des exigences. Dans ce travail, une étude numérique, en utilisant le logiciel ANSYS/FLUENT, de l'écoulement transsonique bidimensionnel sur un profil d'aile NACA0012 a été menée et comparée aux données expérimentales fournies. Ensuite, un algorithme d'optimisation multiobjectif, basé sur l'algorithme génétique NSGA-II (Non-dominated Sorting Genetic Algorithm), a été couplé à ANSYS/FLUENT afin d'obtenir un meilleur contrôle des performances des coefficients aérodynamiques du profil.

ABSTRACT. Multidisciplinary optimization techniques become more and more applied in the field of aerodynamics due to the rapid development of computers high-performance, numerical methods and optimization algorithms. These techniques coupled with Computational Fluid Dynamics (CFD), which aims to incorporate mathematical relations and algorithms to analyze and solve fluid flow problems, involve the use of those numerical methods and algorithms to improve the fluid flow solutions. CFD analysis of an airfoil determines its ability by producing results such as lift and drag forces, and the application of an optimization algorithm involves manipulating these aerodynamic performances according to the requirements. In this work, a numerical investigation, using ANSYS/FLUENT software, of two-dimensional transonic flow over a NACA0012 airfoil was conducted and compared with the provided experimental data. Then a multiobjective optimization algorithm, based on a Non-dominated Sorting genetic algorithm (NSGA-II), was coupled with FLUENT in order to obtain an improved performance control of the aerodynamic coefficients of the airfoil.

MOTS-CLÉS. Aérodynamique, Nombre de Mach, CFD, Optimisation, NSGA-II.

KEYWORDS. Aerodynamic, Mach number, CFD, Optimization, NSGA-II.
\end{abstract}

\section{Introduction}

La nécessité de disposer de méthodes plus rapides et plus précises pour le calcul des champs de flux autour de configurations d'intérêt technique implique l'évolution rapide de la CFD. Dans le passé, la CFD était la méthode de choix pour la conception de nombreux composants et processus aérospatiaux, automobiles et industriels dans lesquels les flux de fluides ou de gaz jouent un rôle majeur. CFD traite de l'analyse numérique de différents phénomènes. Aujourd'hui, des progiciels commerciaux sont disponibles, ce qui fait de la CFD une composante importante de la pratique de l'ingénierie dans les organisations industrielles et environnementales.

Dans le domaine aéronautique, les performances d'une aile d'avion dépendent des caractéristiques de l'écoulement environnant. En effet, la quantification et l'optimisation aérodynamique des ailes d'un avion sont devenues nécessaires pour le contrôle de ces caractéristiques aérodynamiques. L'aérodynamique est définie comme la science qui traite les mouvements et les forces autour d'une structure, où le nombre de recherches augmente rapidement en raison de l'évolution rapide de la CFD [BLO 12, ELM 19]. Ces écoulements de fluides sont soumis à des contraintes visqueuses et à une 
inertie qui produisent des fluctuations désordonnées. La turbulence affecte donc le comportement de l'écoulement aérodynamique ainsi que la structure interagissant avec le fluide dans une plage de Reynolds élevée. Les simulations numériques, dans ce cas, ont été motivées par le besoin de méthodes plus rapides et plus précises pour calculer et analyser l'effet des différents paramètres impliqués dans les performances aérodynamiques [ELH 17, ELM 19].

Dans cet article, une simulation numérique d'un écoulement transsonique bidimensionnel sur un profil NACA 0012 a été traitée en utilisant ANSYS/FLUENT. Ensuite, un algorithme d'optimisation multi-objectifs, basé sur un algorithme génétique de tri non-nominé II (NSGA-II), a été réalisé pour contrôler les coefficients aérodynamiques. La section 2 présente les équations de base et la formulation mathématique de la description de l'écoulement des fluides et du modèle de turbulence utilisés dans notre étude. La section 3 présente quelques concepts de base de l'optimisation multiobjectifs et de l'algorithme génétique de tri non-dominé (NSGA-II). La section 4 présente l'énoncé du problème, les conditions aux limites et les résultats de la comparaison avec les données expérimentales fournies et du processus d'optimisation. Enfin, les principales conclusions sont tirées dans la section 5 .

\section{Formulation mathématique}

\subsection{Mécanique des fluides}

Pour la description des écoulements de fluides, on utilise généralement la formulation eulérienne, car on s'intéresse généralement aux propriétés de l'écoulement à certains endroits dans le domaine de l'écoulement. Nous nous limitons au cas des fluides isotropes linéaires visqueux connus sous le nom de fluides newtoniens, qui sont de loin les plus importants pour les applications pratiques. Les fluides newtoniens sont caractérisés par la loi des matériaux suivante pour le tenseur de contrainte de Cauchy $\mathrm{T}$ :

$$
T_{i j}=\mu\left(\frac{\partial v_{i}}{\partial x_{j}}+\frac{\partial v_{j}}{\partial x_{i}}-\frac{2}{3} \frac{\partial v_{k}}{\partial x_{k}} \delta_{i j}\right)-p \delta_{i j}
$$

avec le vecteur vitesse $\mathrm{v}_{\mathrm{i}}$ par rapport à la coordonnée cartésienne $\mathrm{x}_{\mathrm{i}}$, la pression $\mathrm{p}$, la viscosité dynamique $\mu$, et le symbole de Kronecker $\delta_{\mathrm{ij}}$. Les équations de Navier-Stokes des écoulements incompressibles peuvent être écrites sur le domaine de la mécanique spatiale des fluides comme :

$$
\begin{aligned}
& \frac{(\partial v)}{\partial t}+\nabla \cdot(\rho v \otimes v-\sigma)-\rho f=0 \\
& \nabla \cdot v=0
\end{aligned}
$$

où $\rho, \mathrm{v}$, et $\mathrm{f}$ sont respectivement la densité, la vitesse et la force externe et $\sigma$ le tenseur de contrainte est défini comme :

$$
\sigma(v, p)=-p I+2 \mu \varepsilon(v)
$$

ici, p est la pression, I est le tenseur d'identité, $\mu$ est la viscosité dynamique et $\varepsilon$ (v) est le tenseur de taux de contrainte donné par :

$$
\varepsilon(v)=\frac{1}{2}\left(\nabla v+\nabla v^{T}\right)
$$

Les conditions limites associées au domaine des fluides sont :

$$
\left.v\right|_{\Gamma_{v}}=\bar{v}
$$


où $\bar{v}$ peut être un profil de vitesse connu à la frontière.

\subsection{Modèle de turbulence}

En général, les modèles de turbulence tentent de modifier les équations de Navier-Stokes instables d'origine en introduisant des quantités moyennes et fluctuantes pour produire les équations de NavierStokes moyennes de Reynolds (RANS). Les modèles de turbulence basés sur les équations RANS sont connus sous le nom de modèles de turbulence statistiques en raison de la procédure de moyenne statistique utilisée pour obtenir les équations. Dans notre étude, le modèle k- $\omega$ SST sera utilisé [LAU 72].

\section{Algorithme Génétique de Tri Non-dominé}

Pour différents problèmes de conception, il est souhaitable de parvenir à l'optimisation simultanée d'objectifs multiples [KON 06]. C'est pourquoi, au lieu de chercher une seule solution optimale, un MOGA est nécessaire pour trouver un ensemble de solutions optimales (généralement connues sous le nom de solutions Pareto-optimales). Dans cette étude, l'algorithme MOGA est utilisé pour trouver les solutions Pareto-optimales au problème de l'optimisation de la voilure. L'algorithme génétique de tri non-dominé (NSGA-II), bien connu, est utilisé [DEB 02, DEB 04, SMA 19].

Le NSGA-II commence par une génération aléatoire d'une population initiale $\mathrm{P}_{0}$ de $\mathrm{N}$ individus parents. À la génération $t$, une population $Q_{t}$ de $N$ enfants est créée à partir de la population parent $P_{t}$ en utilisant les opérateurs génétiques (sélection - croisement - mutation). Ensuite, les deux populations sont combinées pour former une nouvelle population $\mathrm{R}_{t}$ de taille $2 \mathrm{~N}$. La recherche des solutions nondominées permet de classer les individus de $\mathrm{R}_{\mathrm{t}}$ en plusieurs fronts de rangs différents. Elle est effectuée de la manière suivante. Chaque individu de $\mathrm{R}_{\mathrm{t}}$ est comparée à tous les autres individus par le concept de dominance. Les individus non-dominés appartiennent au front de rang 1, le front de Pareto. En éliminant, temporairement, ces individus de l'ensemble de recherche, l'algorithme est itéré pour fournir le front de rang 2, et ainsi de suite. La nouvelle population parent $\mathrm{P}_{\mathrm{t}+1}$ est alors construite avec les $\mathrm{N}$ individus appartenant aux fronts de rang les plus faibles. Pour le dernier front, il y a plus de solutions que de places restantes dans la nouvelle population $\mathrm{P}_{\mathrm{t}+1}$. Les individus sont alors triés selon leur distance d'encombrement [DEB 04], et par ordre croissant. Ce choix permet d'offrir la meilleure distribution des individus sur le front de rang le plus élevé.

Lorsque tous les objectifs individuels de la génération actuelle sont calculés, le code MOGA les rassemble et détermine la qualité de chaque individu. Après avoir passé en revue toutes les procédures d'optimisation de l'algorithme génétique décrites dans la section précédente, une nouvelle génération est créée et toutes les étapes sont répétées pour la nouvelle génération. L'algorithme se poursuit jusqu'à ce que la convergence des valeurs objectives soit atteinte.

\section{Simulation numérique}

\subsection{Problématique}

Le profil NACA 0012, bien documenté, a été utilisé. Les simulations ont été évaluées pour un nombre de Mach $\mathrm{M}=0,7$, identique aux données expérimentales fiables [COA 87], afin de valider la présente simulation. La température totale du flux libre est de $284 \mathrm{~K}$, ce qui est identique à la température ambiante. Pour ce nombre de Mach, le flux peut être décrit comme compressible. La viscosité est de $\mu=1,7894.10^{-5} \mathrm{~kg} / \mathrm{m} . \mathrm{s}$ et la densité à la température donnée est de $\rho=1.225 \mathrm{~kg} / \mathrm{m}^{3}$. Un solveur implicite séparé a été utilisé dans FLUENT et les calculs ont été effectués pour l'angle d'attaque $\alpha=1,55^{\circ}$. Les maillages du profil de l'aile ont été créés en utilisant une variété d'éléments quadrilatéraux. La résolution du maillage était plus élevée là où une plus grande précision de calcul est nécessaire, comme le montre la figure 1. 


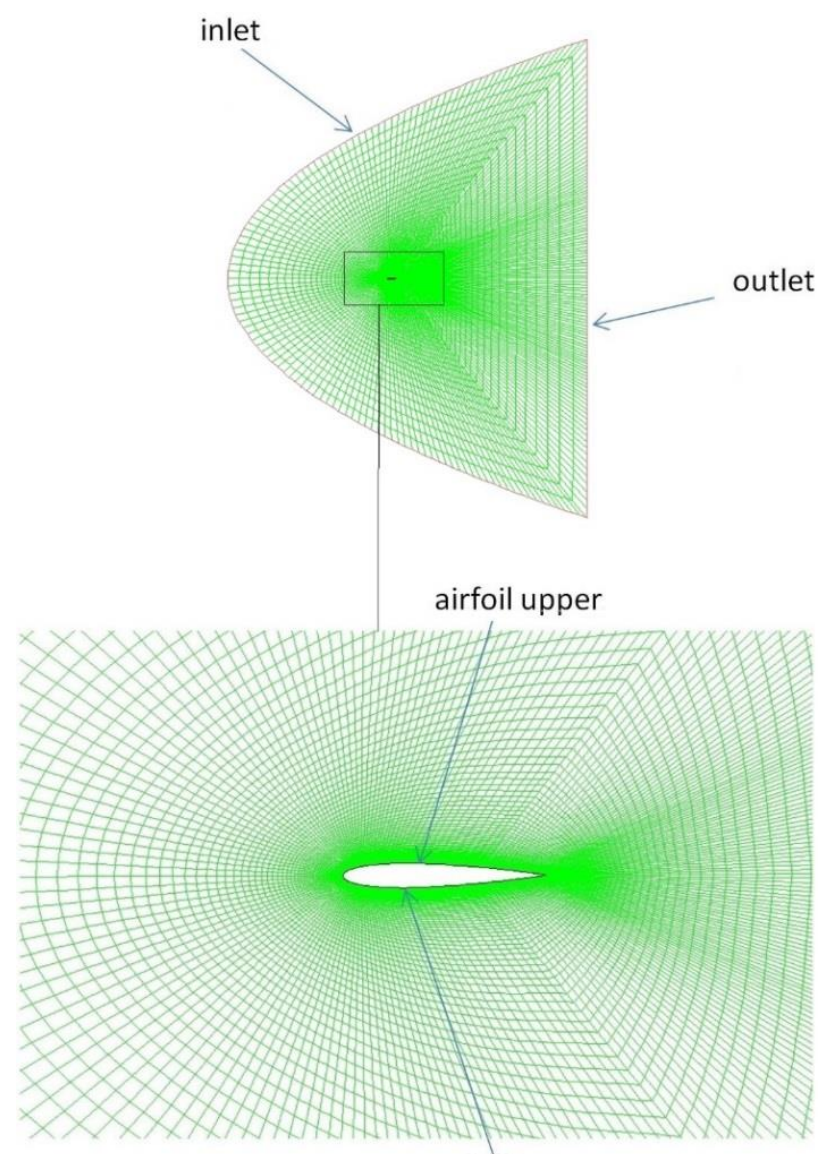

airfoil lower

Figure 1. Conditions aux limites.

\subsection{Résultats de la CFD}

La principale source de données expérimentales, utilisées ici à des fins de comparaison, sont les paramètres d'écoulement mesurés sur des ailes droites, avec des sections transversales inchangées le long de leur envergure, et recueillis dans la base de données expérimentales pour l'évaluation des programmes informatiques (AGARD-AR-138, 1979) pour vérifier les méthodes de calcul de la dynamique des fluides.

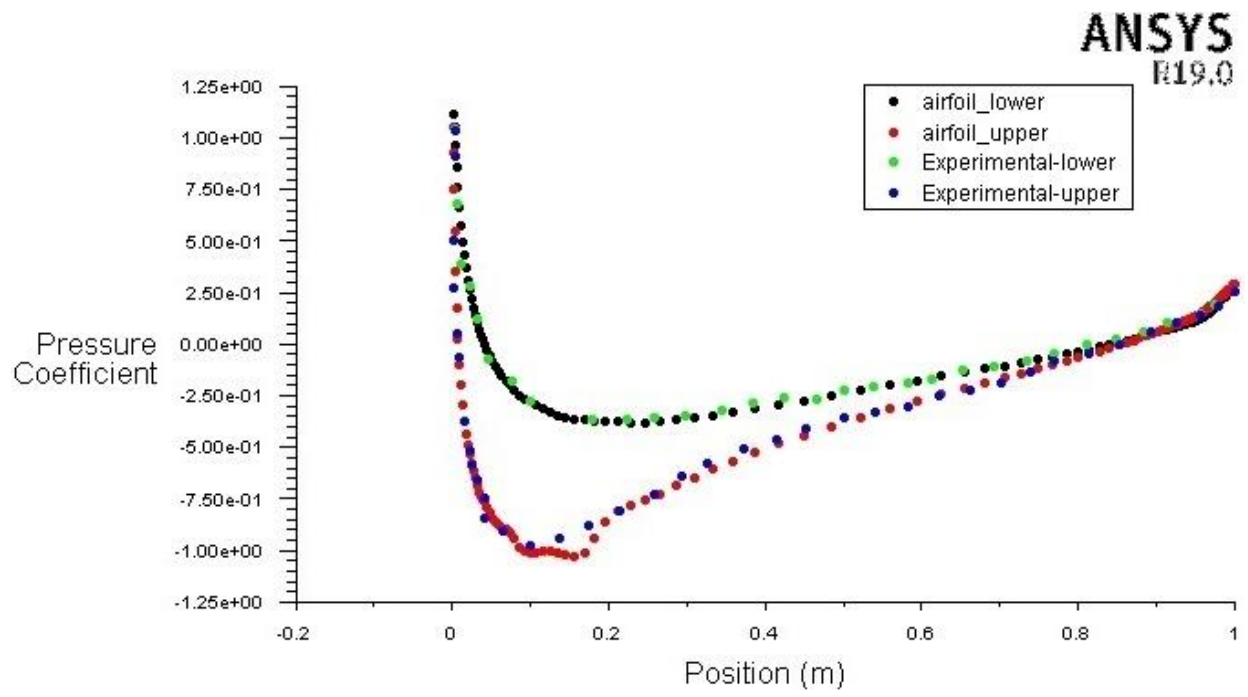

Figure 2. $C_{p}$ le long de l'intrados et l'extrados pour Mach 0.7 

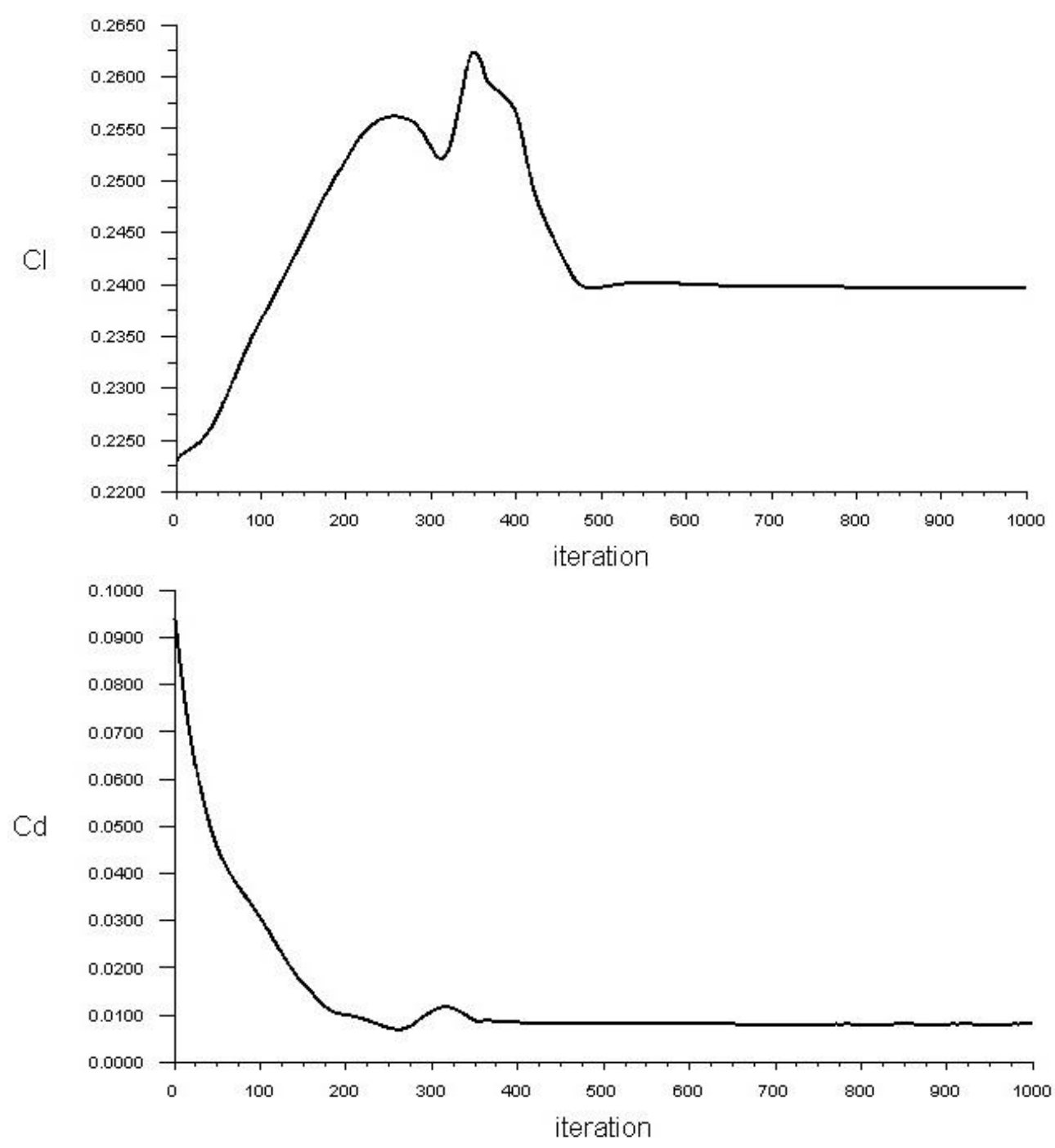

Figure 3. Évolution des coefficients de portance et de traînée.

Selon Hoist (1987) [HOI 87], il est préférable de prendre les données des essais de Harris dans la soufflerie à pression transsonique de 8-pieds, même si les données sur l'écoulement au-dessus de la voilure sont obtenues dans plusieurs souffleries. Les calculs ont été effectués à un nombre de Reynolds de $\operatorname{Re}=9.10^{6}$. La comparaison des résultats calculés avec les données de Harris pour le coefficient de pression est présentée sur la figure 2 à $\mathrm{M}=0.7$ et $\alpha=1.55^{\circ}$. La figure 3 montre l'évolution des cœefficients de portance et traînée calculées sous les mêmes conditions [ELM 19].

\subsection{Optimisation}

Les objectifs de cette optimisation sont d'obtenir un coefficient de traînée minimal avec un coefficient de portance maximal qui conduit à un rapport de finesse aérodynamique maximal, implicitement donné comme ci-dessous :

$$
\left\{\begin{array}{cc}
\min _{d . v}: & f_{1}=C_{d} \\
\max _{d . v}: & f_{2}=C_{l} \\
\text { sous contrainte }: & C_{d}>0
\end{array}\right.
$$

Deux paramètres d'entrée sont utilisés pour le processus d'optimisation, à savoir le nombre de Mach et l'incidence, comme illustré dans le tableau 1 .: 


\begin{tabular}{|c|c|c|c|}
\hline Paramètre d'entrée & Valeur Initiale & Borne inférieure & $\begin{array}{c}\text { Borne } \\
\text { supérieure }\end{array}$ \\
\hline Nombre de Mach & 0.7 & 0.3 & 0.8 \\
\hline Angle d'incidence & 1.55 & 0 & 6 \\
\hline
\end{tabular}

Tableau 1. Paramètres d'entrée de l'aile

\subsubsection{Processus de couplage FLUENT et MOP}

Le travail d'optimisation a été entièrement automatisé via un script principal écrit en MATLAB. Le script démarre le processus d'optimisation multi-objectifs en générant une population initiale à l'aide d'une fonction de distribution aléatoire uniforme. Ensuite, le script principal soumet plusieurs ensembles de variables de décision (c'est-à-dire plusieurs géométries) et attend les valeurs de chaque fonction objectif individuelle avant de poursuivre. MATLAB fait appel au logiciel FLUENT pour modifier les paramètres d'entrée afin de calculer les réponses aérodynamiques prises comme valeurs objectives de la nouvelle forme et renvoie ces valeurs au processus d'optimisation pour exécuter l'algorithme multi-objectif et afficher les résultats optimaux.

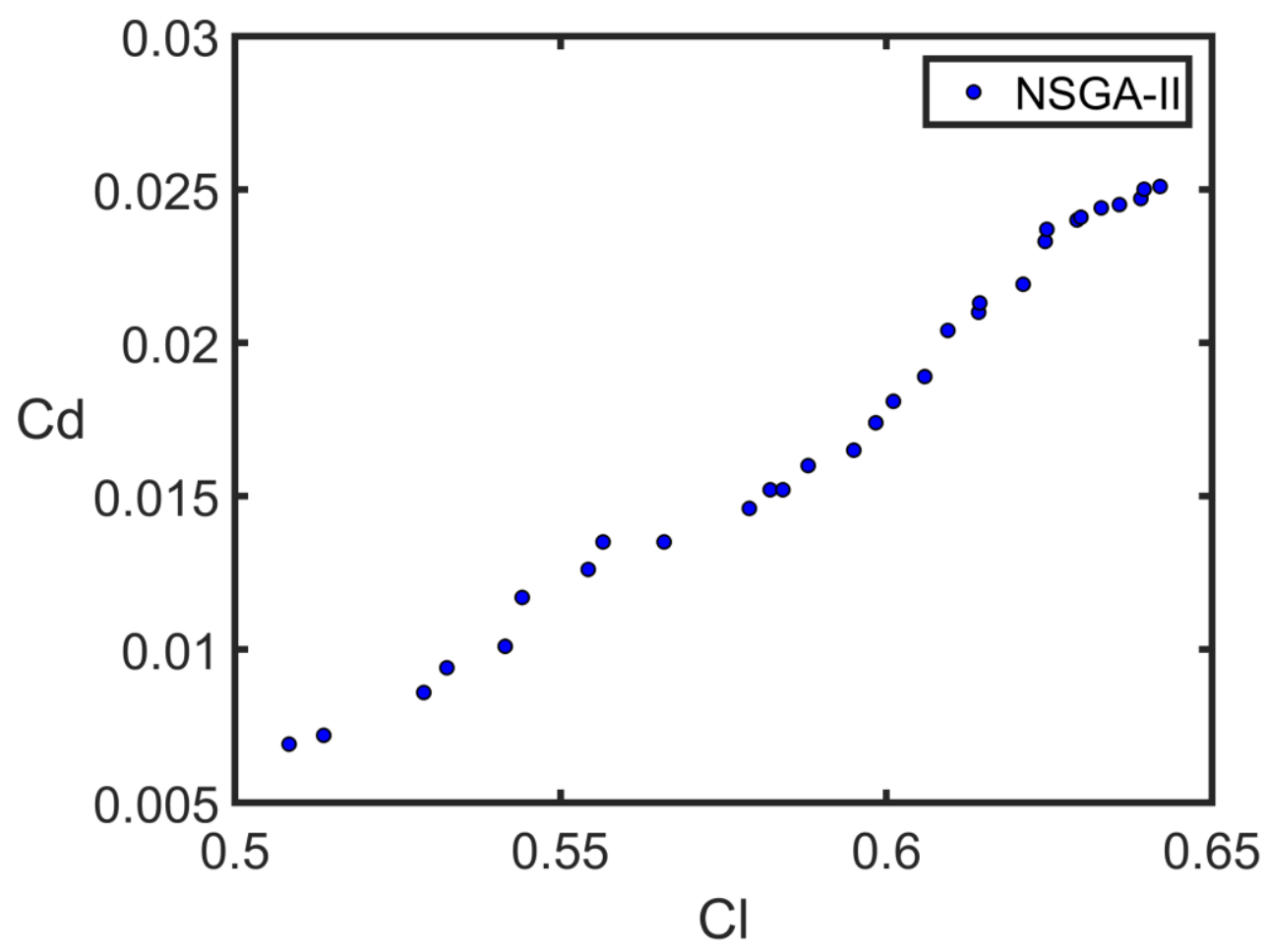

Figure 4. Front de Pareto pour le profil NACA0012

\subsubsection{Résultats de l'optimisation}

L'optimisation multi-objectifs dans cette partie est effectuée pour le profil NACA0012 en utilisant l' algorithme NSGA-II couplés au solveur CFD selon le processus d'optimisation. Le front de Pareto obtenu pour l'algorithme NSGA-II, pour une taille de population et un nombre maximum de générations fixés respectivement à 30 et 30 , est montré sur la figure 4 et les résultats du tableau 2 présentent les meilleures valeurs optimales des fonctions objectives trouvées. 


\begin{tabular}{|c|c|c|c|c|}
\hline & \multicolumn{2}{|c|}{ Variables d'entrée } & \multicolumn{2}{c|}{ Fonctions objectif } \\
\cline { 2 - 5 } & Nombre de Mach & Angle d'incidence & $\mathbf{C}_{\mathbf{d}}$ & $\mathbf{C}_{\mathbf{l}}$ \\
\hline \multirow{2}{*}{ NSGA-II } & 0.581 & 5.999 & $\mathbf{0 . 0 0 6 9}$ & 0.5083 \\
\cline { 2 - 5 } & 5.676 & 0.675 & 0.0251 & $\mathbf{0 . 6 4 2 0}$ \\
\hline
\end{tabular}

Tableau 2. Résultats optimaux de l'aile

\section{Discussion}

La figure 2 montre qu'il n'y a pas de séparation, et que les distributions de pression calculées sont en bon accord avec les distributions expérimentales. La présence d'une onde de choc faible est indiquée dans les calculs près de l'emplacement dans le sens de la corde de $\mathrm{x} / \mathrm{c}=0,15$ et elle n'est pas apparente dans les distributions de pression expérimentales.

Pour les résultats de l'optimisation, le tableau 2 montre les coefficients de portance et de traînée aux conditions d'écoulement citées, obtenus en utilisant la NSGA-II. Par rapport au profil d'origine, les résultats optimisés ont généralement des performances supérieures en matière de réduction de la traînée de choc. De plus, on peut observer qu'un bon $C_{1}$ a été maintenu après l'élimination des chocs, dans le profil optimisé. Les résultats du tableau 2 montrent également que les résultats optimisés ont beaucoup amélioré le rapport $\mathrm{C}_{\mathrm{l}} / \mathrm{C}_{\mathrm{d}}$, passant de 20 à $30 \%$ par rapport à l'aileron d'origine.

\section{Conclusion}

Dans ce travail, ANSYS/FLUENT a été utilisé dans le cadre d'un projet d'établi pour calculer l'écoulement compressible et transsonique au-dessus d'un profil NACA0012. Une comparaison a été faite entre les résultats calculés et les données expérimentales publiées et un bon accord a été obtenu. D'un autre côté, MATLAB a été utilisé pour réaliser un algorithme d'optimisation multi-objectifs basé sur un algorithme génétique de tri non-dominé (NSGA-II), cette étude a apporté plusieurs contributions importantes à l'étude de l'optimisation de la voilure. Il est démontré que la technique NSGA-II peut être utilisée de manière efficace et précise pour produire des résultats aérodynamiques globalement optimaux en utilisant une valeur objective souhaitée $\left(C_{1}, C_{d}\right.$ et/ou $\left.C_{1} / C_{d}\right)$ extraite d'une analyse CFD.

\section{Bibliographie}

[BlO 12] Blocken B., Van Druenen T., Toparlar Y., Malizia F., Mannion T., Andrianne T., Marchal T., MAAS G., DiEPENS J., «Aerodynamic drag in cycling pelotons: new insights by CFD simulation and wind tunnel testing », Journal of Wind Engineering and Industrial Aerodynamic, 179: 319-337, 2012.

[ELM 19] El MaAni R., RADI B., Al HAMI A., « Multiobjective backtracking search algorithm: application to FSI», Structural and Multidisciplinary Optimization, 59(1): 131-151, 2019.

[ELH 17] El HAMi A., RADI B., «Fluid-Structure Interactions and Uncertainties: Ansys and Fluent Tool», $P$ John Wiley and Sons, 2017.

[ELM 19] El MAANi R., RADI B., Al HAMI A., « CFD Analysis and Shape Optimization of NACA0012 Airfoil for Different Mach Numbers », Proceedings of the 5th International Conference on Optimization and Applications (ICOA), 2019.

[LAU 72] Launder B. E., Spalding D. B., «Lectures in Mathematical Models of Turbulence », Academic Press, London, England, 1972.

[FER 96] Ferziger J. H., Peric M., «Computational Methods for Fluid Dynamics », Springer, Berlin, Germany, 1996.

[COA 87] COAKLEY T. J., « Numerical Simulation of Viscous Transonic Airfoil Flows», NASA Ames Research Center, AIAA-87-0416, 1987. 
[HOI 87] HOIST T. L., « Viscous transonic airfoil workshop compedium of results », AIAA Paper No. 87-1460, 1987.

[HAR 81] HARRIS C. D., «Two-Dimensional Aerodynamic Characteristics of the NACA 0012 Airfoil in the Langley 8-foot Transonic Pressure Tunnel », NASA Ames Research Center, NASA TM 81927, 1981.

[KON 06] KonaK A., COIT D. W. AND SMITH A. E., « Multi-objective optimization using genetic algorithms: a tutorial », Reliabil. Eng. Syst. Safety, 9: 992-1007, 2006.

[DEB 02] Deb K., Pratap A., Agarwal S., «A fast and elitist multi-objective genetic algorithm: NSGA-II », IEEE Trans. Evol. Comput., 6(2): 182-197, 2002.

[DEB 04] DEB K., « Single and multi-objective optimization using evolutionary computation », In Proceedings of the 6th international conference on hydroinformatics, Singapore, pp. 14-35, 2004.

[SMA 19] SMAOUI, H., MAQSOUD, A., KAIDI, S., "Transmissivity Identification by Combination of CVFEM and Genetic Algorithm: Application to the Coastal Aquifer ». Mathematical Problems in Engineering, 3463607, 2019. 